\title{
Ultrafast collective dynamics of short-range charge/orbital ordering fluctuations in colossal magnetoresistive oxides
}

\author{
Y. H. Ren ${ }^{1}$, Y. F. Hu ${ }^{2}$, Qi Lì ${ }^{2}$, C. S. Hong ${ }^{3 \dagger}$, N. H. Hur ${ }^{3}$, and G. Lüpke ${ }^{1}$ \\ ${ }^{1}$ Applied Science, the College of William \& Mary, \\ Williamsburg, VA 23187 \\ ${ }^{2}$ Department of Physics, Pennsylvania State University, University Park, \\ $P A 16802$ \\ ${ }^{3}$ Center for CMR Materials, Korea Research Institute of Standards and \\ Science, P. O. Box 102 Yusong, Daejeon, 305-600, Republic of Korea
}

(November 13, 2018)

\begin{abstract}
The "colossal magnetoresistive" (CMR) manganites are highly correlated systems with a strong coupling between spin, charge, orbital, and lattice degrees of freedom, which leads to complex phase diagrams and to the coexistence of various forms of ordering. For example, nanoscale charge/orbital ordering (CO) fluctuations appear to cooperate with Jahn-Teller (JT) distortions of the $\mathrm{MnO}_{6}$ octahedra in CMR manganites and compete with the electron itinerancy favored by double exchange. However, access to the ordered dynamical state has been challenging, mostly due to intrinsic experimental difficulties in measuring fast short-range correlations. Here, we report on a strongly damped low-energy collective mode originating from fast short-range $\mathrm{CO}$ fluctuations in $\mathrm{La}_{0.67} \mathrm{Ca}_{0.33} \mathrm{MnO}_{3}$ (LCMO) single crystal and thin films. We elucidate the collective mode in terms of its dispersion relation and dependence on average
\end{abstract}




\section{A-site ion radius, $r_{A}$, and hole-doping concentration. Our results show for the first time that dynamical short-range CO correlations in CMR manganites can be detected with high momentum resolu- tion by coherent ultrafast optical techniques.}

Charge/orbital ordering $(\mathrm{CO})$ is a common characteristic of transition metal oxides with perovskite structure [1-4]. Recent neutron scattering experiments showed that fast shortrange correlation of the so-called CE-type charge ordering exists in the colossal magnetoresistive (CMR) manganite, LCMO, above the Curie temperature, $T_{C}[5,6]$. The coupled dynamics of fluctuating CO phases and Jahn-Teller (JT) distortions of the $\mathrm{MnO}_{6}$ octahedra are found to be crucial for the metal-insulator (MI) phase transition and the CMR effect $[7,8]$. Therefore, it is fundamentally important to elucidate the dynamics of short-range charge/orbital stripes in CMR materials.

Ultrafast optical techniques have provided significant insight into coupled electron, lattice and spin dynamics in metals [9,10], and more recently, transition metal oxides [11-15]. In this work, we investigate for the first time the collective dynamics of short-range $\mathrm{CO}$ fluctuations in CMR manganites by time-resolved optical spectroscopy. The period of the $\mathrm{CO}$ modulations show strong dependence on hole doping concentration and average A-site ion radius, which is explained by different correlation length and strength in these materials. We obtain a coherence length of $\sim 250 \mathrm{~nm}$ for LCMO and $>>2.5 \mu \mathrm{m}$ for $\mathrm{LaMnO}_{3}$ (LMO). The overdamped behavior in LCMO is due to inhomogeneous dephasing, whereas in LMO, the damping rate is very small because of the static and long-range correlation. Our results show that the time-resolved optical technique provides momentum-resolved spectroscopic information on the low-energy dynamics of short-range CO fluctuations in colossal magneto-resistive materials. This important new information is relevant for elucidation of collective transport phenomena in strongly correlated electron systems.

In the time-resolved optical experiments, the change of reflectivity $(\Delta R)$ induced by the pump beam is measured as a function of time delay for different wavelengths of the probe beam $\left(\lambda_{\text {probe }}=400 \mathrm{~nm}-2.5 \mu \mathrm{m}\right)$ in both LCMO single crystal and thin films. Figure 
1 shows typical time evolution of $\Delta R$ for LCMO thin film and LMO single crystal as a function of time delay $\Delta t$ between the pump and probe pulses. The data are taken at 285 $\mathrm{K}$ and $35 \mathrm{~K}$ with pump and probe wavelength of $800 \mathrm{~nm}$, respectively. After the initial laser pulse excitation, the decay of $\Delta R$ clearly shows an oscillatory component on top of a multi-exponential decay.

Here, we discuss only the slow oscillatory component observed in the trace of $\Delta R$ from LCMO and LMO (Fig. 1). Figure 2 displays the coherent overdamped oscillations of $\Delta R$ from LCMO thin film without the exponentially decaying part as a function of probe wavelength in the range $400 \mathrm{~nm}$ to $2300 \mathrm{~nm}$. The oscillations are heavily damped and strongly dispersive. Only one, at most two oscillations, are observed in the whole wavelength range. The frequency of the oscillations decreases from $73.83 \mathrm{GHz}$ at $400 \mathrm{~nm}$ to $21.8 \mathrm{GHz}$ at $2300 \mathrm{~nm}$.

Figure 3 shows the dispersion relation of the slow oscillation of $\Delta R$ from LCMO thin film at $285 \mathrm{~K}$. The wave number is given by $q=2 n / \lambda$, where $\lambda$ is the probe wavelength and $n$ is the refractive index of LCMO [16]. The frequency of the coherent oscillations increases proportional to the wave number of the excited mode. The slope gives a phase velocity for the collective mode of $c_{\phi}=7.1 \pm 0.3 \times 10^{3} \mathrm{~m} / \mathrm{s}$ along the $c$-axis.

The coherent oscillations of $\Delta R$ show strong dependence on hole doping concentration and average $\mathrm{A}$-site ion radius, $r_{A}$. The former is clearly revealed in $\Delta R$ from LMO (Fig. 1). Similar to the LCMO sample, $\Delta R$ also exhibits a slow oscillatory component on top of a bi-exponential decay in the parent compound LMO. However, the 800-nm data from LMO reveal a different modulation period of $\sim 45 \mathrm{ps}$, in contrast to $\sim 27.6$ ps observed in LCMO (Fig. 1). The oscillations also show a strong dependence on A-site substitution (average A-site ion radius, $\left.r_{A}\right)$. The modulation period for PCMO single crystal at $800 \mathrm{~nm}$ is $\sim 40$ ps [17], which is much longer than in LCMO.

These observations strongly suggest that the coherent oscillations originate from a lowenergy collective mode in LCMO. The fact that the period of the oscillations is longer in the parent compound LMO and PCMO, which has a smaller average ionic radius than 
LCMO, can be explained by different correlation length and strength in these materials. As compared to LCMO, where the charge/orbital correlations are short-range in nature due to the presence of ferromagnetic phases, the charge/orbital coupling is stronger and long-range in both LMO and the smaller bandwidth manganite PCMO. The case of LMO, with no double-exchange carriers, is a typical example in which the orbital ordering is enhanced by the increase of Jahn-Teller interaction. The stronger charge/orbital correlation in LMO results in a heavier effective mass than in LCMO. The phase velocity of the collective modes is determined by the effective mass and the Fermi velocity, $v_{F}[18]$ :

$$
c_{\phi}=\left(\frac{m}{m^{*}}\right)^{1 / 2} v_{F}
$$

which is therefore smaller in LMO than in LCMO. We expect a longer oscillation period in LMO since the modulation period is inversely proportional to the phase velocity of the material. Similarly, the ground state of PCMO exhibits charge-localizing real space ordering of $\mathrm{Mn}^{3+}$ and $\mathrm{Mn}^{4+}$ ions that occurs at $\sim 220 \mathrm{~K}$. The charge/orbital correlation in PCMO is enhanced by the increase of orthorhombic distortion of $\mathrm{GdFeO}_{3}$-type [19]. Thus, a longer oscillation period is expected in PCMO as well.

Another particularly intriguing result is the strong doping concentration dependence of the damping rate of the coherent oscillations in LCMO. As shown in Fig. 1, the coherent oscillations in LMO persist for at least 600 ps ( $\sim 15$ periods) with very little damping whereas at most two periods of the collective mode can be observed in LCMO. Since the optical properties of LCMO and LMO are very similar at photon energies in the near infrared to visible range [20,21], the stronger damping observed in LCMO must be directly related to the damping of the collective mode. The results shown in Fig. 1 allow us to extract the coherence length

$$
\lambda=\tau c_{\phi}
$$

for LCMO and LMO, which depends essentially on the damping time $\tau$ of the collective mode. We obtain a coherence length of $\sim 250 \mathrm{~nm}$ for LCMO and $>>2.5 \mu \mathrm{m}$ for LMO. 
The overdamped behavior in LCMO may result from inhomogeneous spatial distribution (inhomogeneous dephasing) and the dynamical nature of the short-range charge/orbital ordering phases. The coherence length for LCMO is of the same order of magnitude as the charge/orbital correlation length of the CE-type phase in perovskite manganites with a characteristic length scale of stripe domains on the order of 100 lattice spacings [22]. In contrast, the damping rate is very small for LMO, because the orbital domains in LMO are static and the correlation is long-range. There is also a very limited phase space available for scattering into other modes in the wavelength range investigated.

The inset of Fig. 1 illustrates the optically excited collective modes in LCMO and LMO. The photo-generated charge/orbital ordering modulations cause complex rotations of the $\mathrm{MnO}_{6}$ octahedra, which oscillate along the modulation direction. The collective modes propagate from the surface in the crystallographic c-direction and approach zero with increasing distance from the origin due to the finite range of correlations.

Finally, we present a simple physical model that accounts correctly for the excitation and detection of the coherent charge/orbital-ordering oscillations. The number of photons per pulse and unit volume absorbed in the sample is $\sim 10^{20}$ photons $/ \mathrm{cm}^{3}$, comparable to the charge-carrier density $\left(\sim 10^{20}-10^{21}\right.$ holes $\left./ \mathrm{cm}^{3}\right)$ in LCMO; hence, one expects significant electron excitation during ultrashort pump pulse illumination. This can lead to coherent excitation of collective modes [23]. We use a tunable optical probe pulse to detect the various frequency components of the photo-generated collective modes. In the back-scattering geometry (Fig. 4), part of the probe pulse is reflected by the wave front of the excited collective modes and the remainder at the surface of the sample. These reflections interfere constructively or destructively depending on the position and time of the charge density modulation. Further, the momentum selection rule for back scattering is $q_{i}+q_{f}=q \cos \theta$, where $q_{i}$ and $q_{f}$ are the wave vectors of the incident and scattered probe beam in the material, and $\theta$ is the probe beam incident angle $\left(\theta \sim 0^{\circ}\right)$. Therefore, for a given probe wavelength phase-matching occurs exclusively for a single collective mode wavevector. This process causes the probe signal to oscillate with time delay relative to the pump pulse. The 
new scheme provides momentum-resolved spectroscopic information of collective modes in solids.

In summary, we investigated fast short-range CO fluctuations in LCMO by time-resolved optical spectroscopy. The amplitude, period and damping of the CO modulations show strong dependence on hole doping and average A-site ion radius. The results reported here have great significance for understanding the competition/cooperation behavior and nanoscale phase separation in colossal magnetoresistive manganites. Our scheme represents a new approach to investigate low-energy collective dynamics in strongly correlated electron systems with high-momentum resolution.

\section{Methods}

LCMO and LMO single crystals and thin films were grown by the floating zone method and pulsed laser deposition. The samples were characterized by electrical resistivity and magnetization measurements. The LCMO single crystal and 400-nm thin film (grown on $\mathrm{NdGaO}_{3}$ (110) substrate) have a Curie temperature $T_{C}=225 \mathrm{~K}$ and $260 \mathrm{~K}$, respectively. The LMO single crystal shows a Neel temperature $T_{N}=145 \mathrm{~K}$. For the transient reflectivity measurements the samples were mounted in an optical cryostat. The laser system consists of a Ti:sapphire regenerative amplifier (Spitfire, Spectra-Physics) and an optical parametric amplifier (OPA-800C, Spectra-Physics) delivering 100-fs short pulses at a 1-kHz repetition rate tunable from $600 \mathrm{~nm}$ to $10 \mu \mathrm{m}$. A two-color pump-probe setup is employed with the pump beam power $<6 \mathrm{~mW}$ and the probe beam power $<1 \mathrm{~mW}$. The unfocused pump beam, spot-diameter $\sim 2 \mathrm{~mm}$, and the time-delayed probe beam are overlapped on the sample with their polarization perpendicular to each other. The reflected probe beam is detected with a photodiode detector. A SR250 gated integrator \& boxcar averager, and a lock-in amplifier are used to measure the transient reflectivity change $\Delta R$ of the probe beam.

$\dagger$ Current address for C. S. Hong, Department of Chemistry, Korea university, Anamdong, Sungbuk-ku, Seoul 136-701, Republic of Korea 


\section{REFERENCES}

[1] Millis, A. J., Lattice effects in magnetoresistive manganese perovskites, Nature 392, 147 (1998).

[2] Coey, J. M. D., Viret, M., and Molnar, S. von, Mixed-valence manganites, Adv. Phys. 48, 167 (1999).

[3] Imada, M. I., Fujimori, A., Tokura, Y., Metal-insulator transitions, Rev. Mod. Phys. 70, 1039 (1998).

[4] Chuang, Y. -D., Gromko, A. D., Dessau, D. S., Kimura, T., and Tokura, Y., Fermi surface nesting and nanoscale fluctuating charge/orbital ordering in colossal magnetoresistive oxides, Science 292, 1509 (2001).112.

[5] Lynn, J. W., et al. Unconventional ferromagnetic transition in $\mathrm{La}_{1-x} \mathrm{Ca}_{x} \mathrm{MnO}_{3}$, Phys. Rev. Lett. 76, 4046 (1996).

[6] Teresa, J. M. De et al., Evidence for magnetic polarons in the magnetoresistive perovskites, Nature 386, 256 (1997).

[7] Dai, Pengcheng, et al. Short-range polaron correlations in the ferromagnetic $\mathrm{La}_{1-x} \mathrm{Ca}_{x} \mathrm{MnO}_{3}$, Phys. Rev. Lett. 85, 2553 (2000).

[8] Adams, C. P., Lynn, J. W., Mukovskii, Y. M., Arsenov, A. A., and Shulyatev, D. A., Charge ordering and polaron formation in the magnetoresistive oxide $\mathrm{La}_{0.7} \mathrm{Ca}_{0.3} \mathrm{MnO}_{3}$, Phys. Rev. Lett. 85, 3954 (2000).

[9] Beaurepaire, E., Merle, J.-C., Daunois, A., and Bigot, J.-Y., Ultrafast spin dynamics in ferromagnetic nickel, Phys. Rev. Lett. 76, 4250 (1996).

[10] Koopmans, B., van Kampen, M., Kohlhepp, J. T., and de Jonge, W. J. M., Ultrafast magneto-optics in nickel: magnetism or optics?, Phys. Rev. Lett. 85, 844 (2000).

[11] Dodge, J. S., et al., Time-resolved optical observation of spin-wave dynamics, Phys. 
Rev. Lett. 83, 4650 (1999).

[12] Kise, T., et al., Ultrafast spin dynamics and critical behavior in half-metallic ferromagnet: $\mathrm{Sr}_{2} \mathrm{FeMoO}_{6}$, Phys. Rev. Lett. 85, 1986 (2000).

[13] Rast, S., et al., Evidence for two coupled subsystems in the superconducting state of $\mathrm{La}_{2-x} \mathrm{Sr}_{x} \mathrm{CuO}_{4}$, Phys. Rev. B 64, 214505 (2001).

[14] Ren, Y. H., Zhao, H. B., Lüpke, G., Hu, Y. F., Li, Qi, Strain-dependent spin dynamics in $\mathrm{Nd}_{0.67} \mathrm{Sr}_{0.33} \mathrm{MnO}_{3}$ near the metal-insulator transition, J. Appl. Phys. 91, 7514 (2002).

[15] Ren, Y. H., et al., Observation of strongly damped GHz phonon-polariton oscillations in $\mathrm{La}_{0.67} \mathrm{Ca}_{0.33} \mathrm{MnO}_{3}$, Phys. Rev. B, 64, 144401 (2001).

[16] Kim, K. H., Jung, J. H., and Noh, T. W., Polaron absorption in a perovskite manganite $\mathrm{La}_{0.7} \mathrm{Ca}_{0.3} \mathrm{MnO}_{3}$, Phys. Rev. Lett. 81, 1517 (1998).

[17] Fiebig, M., Miyano, K., Tomioka, Y., and Tokura, Y., Sub-picosecond photo-induced melting of a charge-ordered state in a perovskite manganite, Appl. Phys. B 71, 211 $(2000)$.

[18] The Fermi velocity $v_{F}$ is similar in LCMO and LMO. See e.g. Singh, D. J., Pickett, W. E., Pseudogaps, Jahn-Teller distortions, and magnetic order in manganite perovskites, Phys. Rev. B 57, 88 (1998).

[19] Coey, J. M. D., BViret, M., Ranno, L., and Ounadjela, K., Electron localization in mixed-valence manganites, Phys. Rev. Lett. 75, 3910 (1995).

[20] Quijada, M., et al., Optical conductivity of manganites: crossover from Jahn-Teller small polaron to coherent transport in the ferromagnetic state, Phys. Rev. B 58, 16093 (1998).

[21] Quijada, M. A., et al., Temperature dependence of low-lying electronic excitations of $\mathrm{LaMnO}_{3}$, Phys. Rev. B 64, 224426 (2001). 
[22] Nelson, C. S., et al., Coherent x-ray scattering from manganite charge and orbital domains, Phys. Rev. B 66, 134412 (2002).

[23] Since the penetration depth, $\xi$, in LCMO is very small $(\sim 100 \mathrm{~nm})$, the momentum bandwidth of the excited coherent collective modes must be large: from $\delta q \delta x>1$ and $\delta x \sim \xi \sim 100 \mathrm{~nm}$ follows that $\delta q>1 \times 10^{5} \mathrm{~cm}^{-1}$, i.e., larger than the biggest $q$ probe used. Hence, we expect to generate a broad band of collective modes, comparable to the entire range of probe wavelengths.

\section{Acknowledgements}

This work is supported in part by NSF through grants: DMR-0137322, IMR-0114124 (CWM), and DMR-9876266 (PSU), and the Petroleum Research Fund (PSU).

Correspondence and requests for materials should be addressed to G. L.

\section{Competing financial interests}

The authors declare that they have no competing financial interests.

\section{Figure captions}

Figure 1. Time evolution of reflectivity change, $\Delta R$, for LCMO thin film at $285 \mathrm{~K}$ and LMO single crystal at $35 \mathrm{~K}$. The transient reflectivity clearly reveals coherent oscillations on top of a multi-exponential decay. The inset shows a schematic illustration of optically excited collective modes in LCMO and LMO. The photo-generated charge/orbital ordering modulations cause complex rotations of the $\mathrm{MnO}_{6}$ octahedra, which oscillate along the modulation direction and approach zero with increasing distance from the origin due to the finite range of correlations. 
Figure 2. a) Time evolution of $\Delta R$ from LCMO thin film for probe wavelengths from ultraviolet $(400 \mathrm{~nm})$ to mid-infrared $(2300 \mathrm{~nm})$; b) Fourier transforms of $\Delta R$ traces shown in a).

Figure 3. Collective mode dispersion relation of LCMO thin film with thickness $400 \mathrm{~nm}$ grown on $\mathrm{NdGaO}_{3}$ (110) substrate at $285 \mathrm{~K}$. Solid squares: the experimental data. Solid line: the predicted dependence $\nu=2 n c_{\phi} \cos \theta / \lambda$, with $\lambda$ the probe wavelength, $n$ the refractive index of LCMO, and $\theta$ the probe beam incident angle $\left(\theta \sim 0^{\circ}\right)$.

Figure 4. Schematic of photo-induced coherent CO mode excitation and detection mechanism. The strong absorption of ultrashort pump pulses generates broad-band coherent CO oscillations. The modes propagate into the sample and are detected in the back-scattering geometry by tunable optical probe pulses. Momentum selection rule for back scattering is $q_{i}+q_{f}=q \cos \theta$, with $q_{i}$ and $q_{f}$ the wave vectors of the incident and scattered probe beam in the material. 


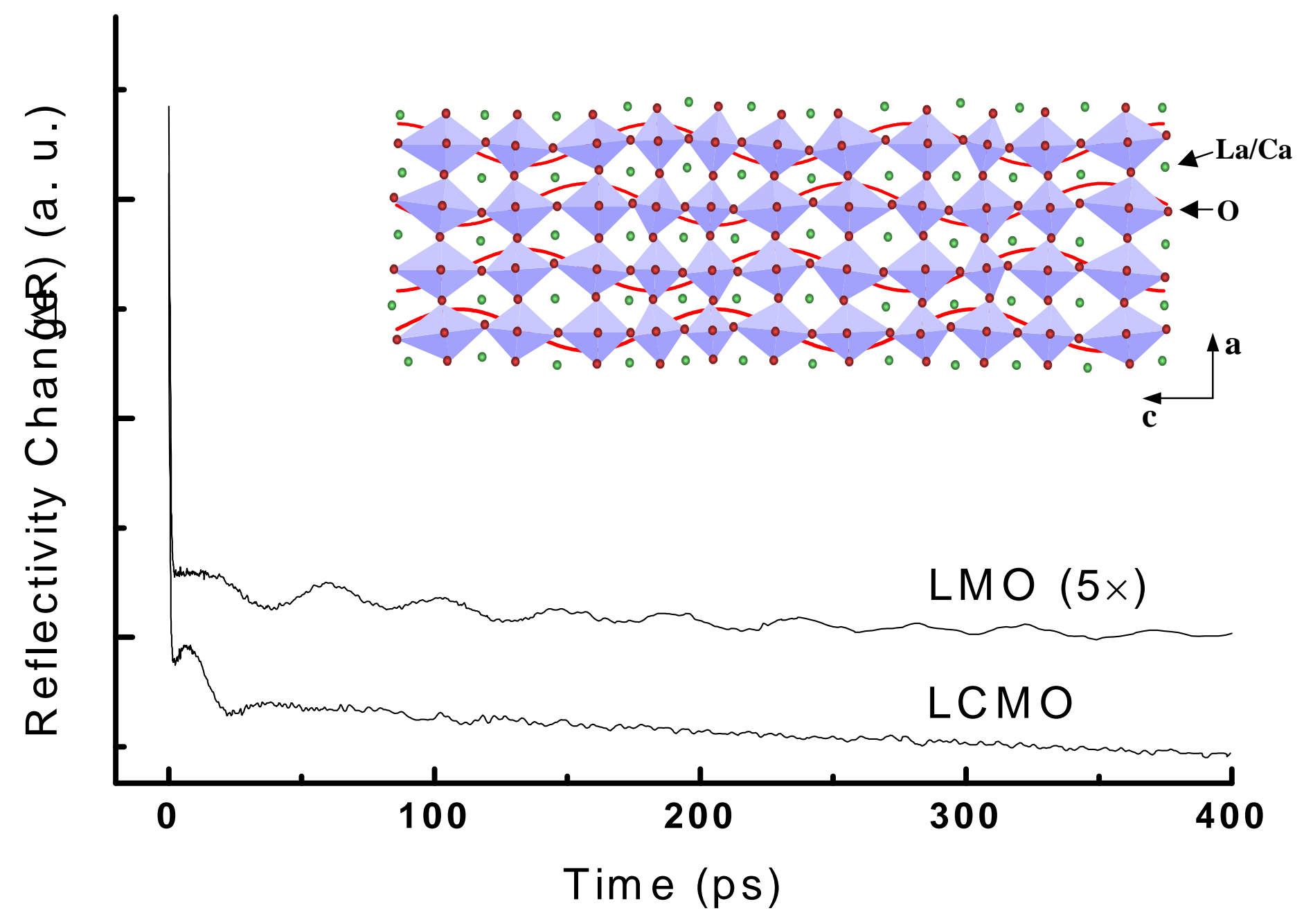

Fig. 1 


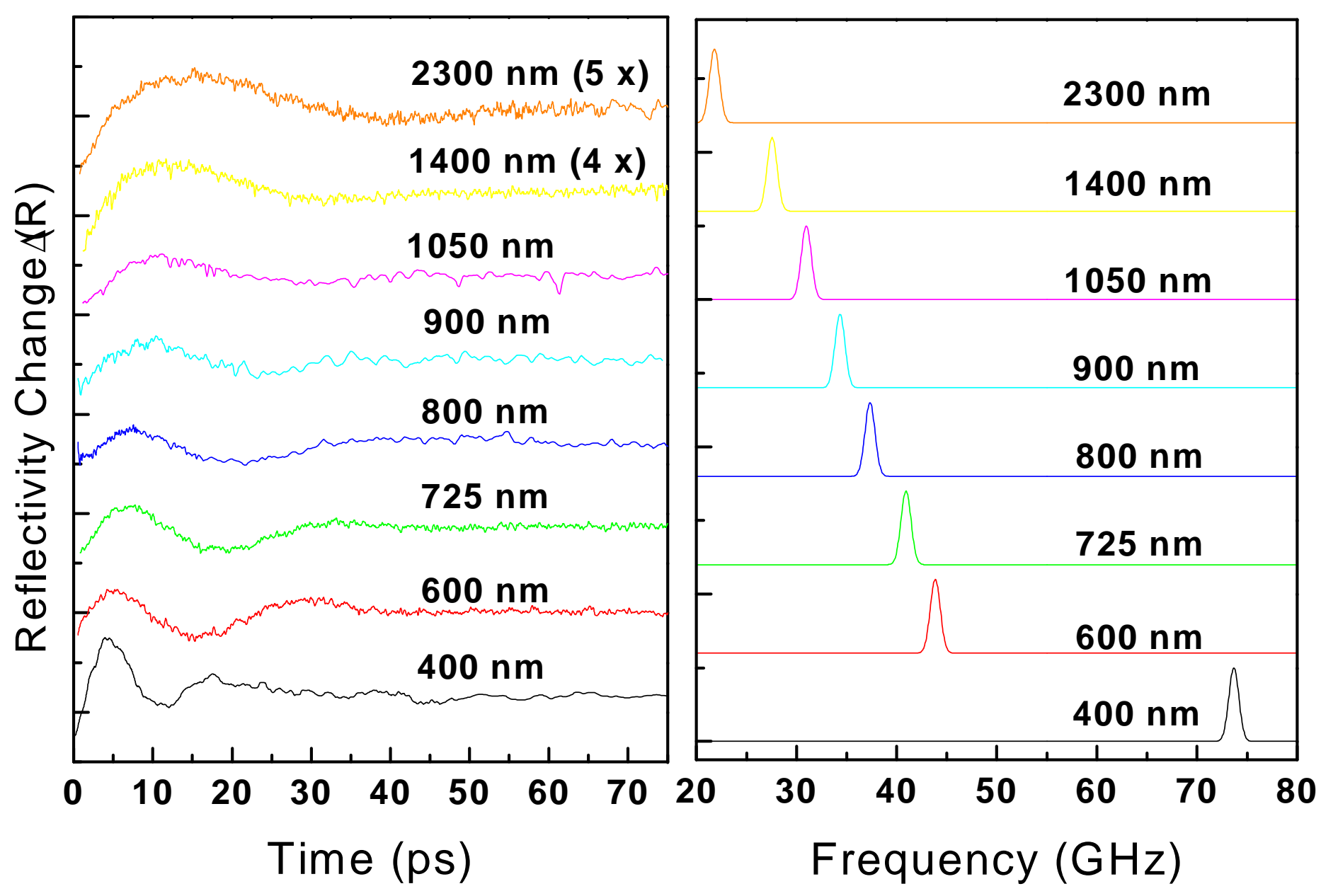

Fig. 2 


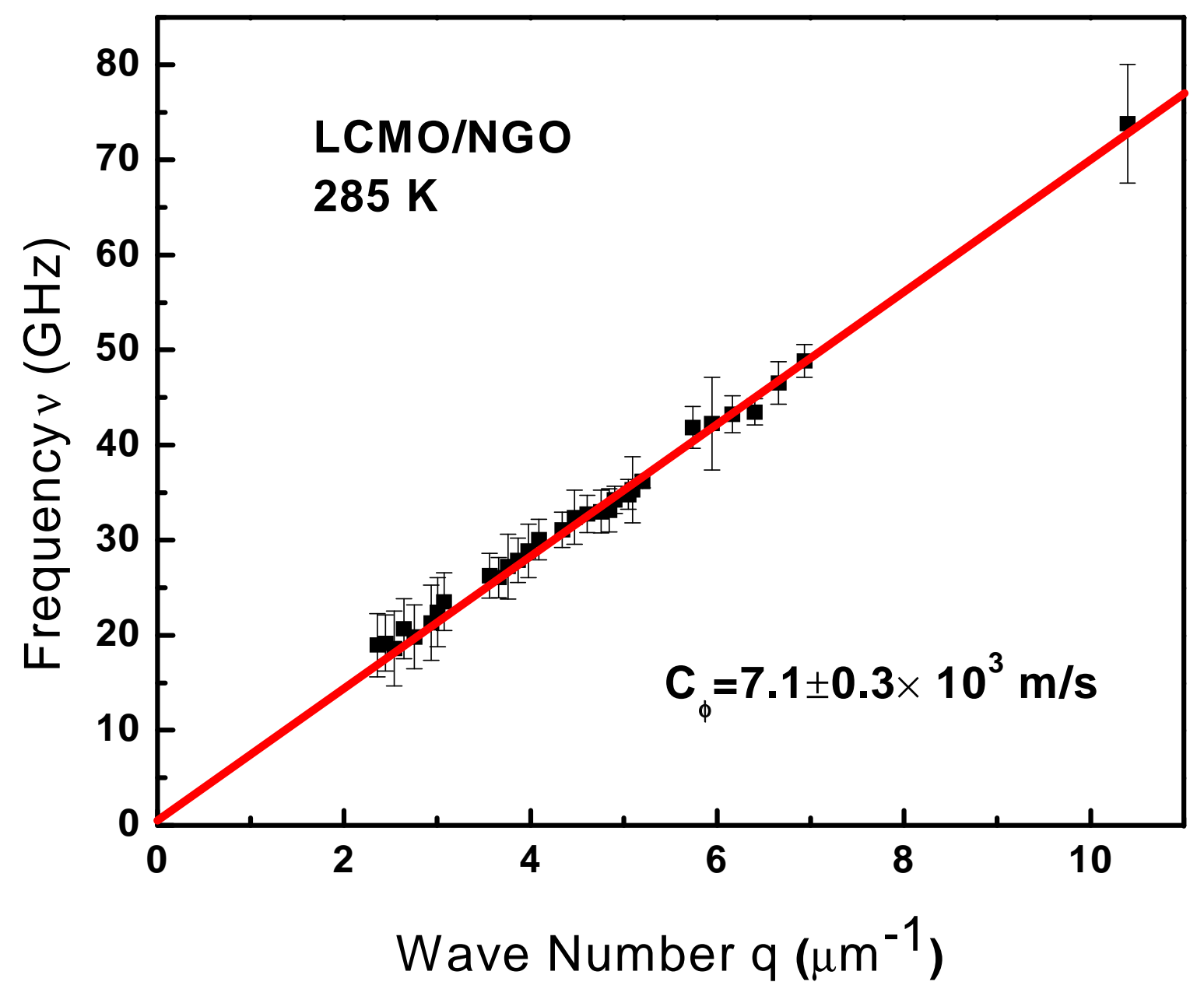

Fig. 3 


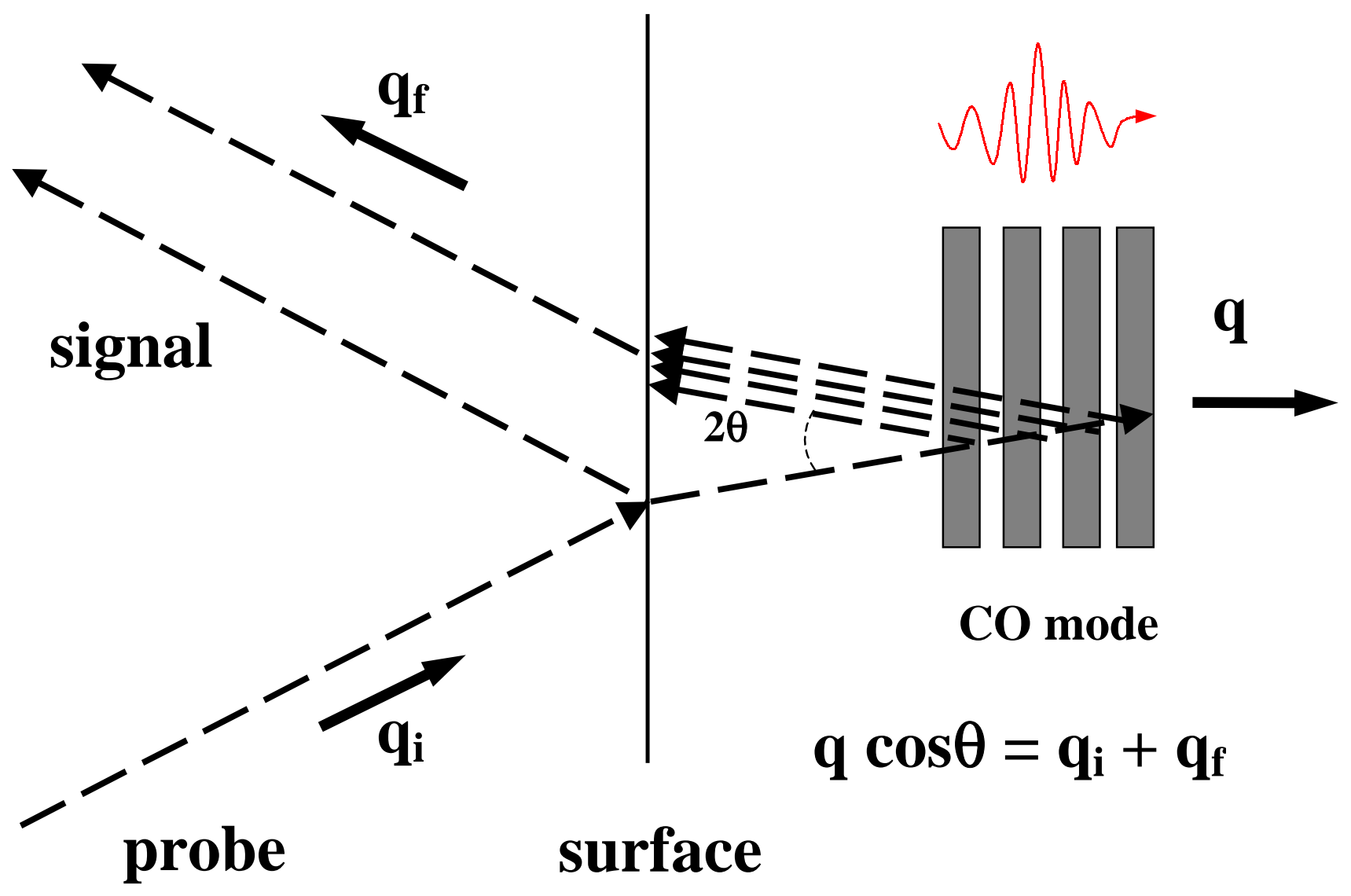

Fig. 4 